Check for updates

Cite this: RSC Adv., 2017, 7, 29722

Received 9th March 2017 Accepted 23rd May 2017

DOI: $10.1039 / c 7 r a 02886 e$

rsc.li/rsc-advances

\section{Recyclable 3D graphene aerogel with bimodal pore structure for ultrafast and selective oil sorption from water $\dagger$}

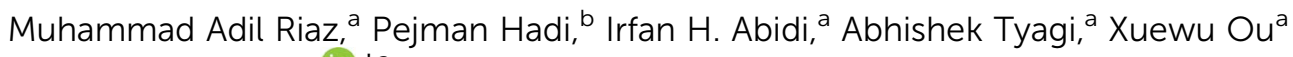 \\ and Zhengtang Luo (iD) *a
}

Development of next-generation porous sorbents to overcome the challenges, such as low uptake capacity, slow sorption rate, and non-recyclability, associated with conventional sorbents is of utmost importance. Herein, we report the synthesis of a highly porous graphene aerogel (GA) with a unique three-dimensional hierarchical bimodal porous network of macro and meso-pores via a facile hydrothermal technique; this aerogel has sorption capacity that is more than 5 times that of conventional commercial sorbents. Fluoroalkyl silane functionalization of the GA surface results in a significant reduction in its water sorption from $20 \mathrm{~g} \mathrm{~g}^{-1}$ to $5 \mathrm{~g} \mathrm{~g}^{-1}$ due to the GA surface becoming more hydrophobic, which renders it useful in practical application to selectively remove oil from seawater. Moreover, the sorption rate of the GA for oils and organic solvents has been found to be extremely fast, and saturation of the GA is completed in a few seconds. This is attributed to its unique meso-macro bimodal porous structure with large pore channels called macro-pores or voids of various sizes ranging from $300 \mathrm{~nm}$ to over $10 \mu \mathrm{m}$, which facilitate mass transport into its inner mesopores of 14-18 $\mathrm{nm}$ at high rate. Finally, the GA is shown to be a highly recyclable material due to its good mechanical strength, where the oil- and organic solvent-sorbed GA can be efficiently recovered using thermal or chemical methods for several sorption-desorption cycles without significant loss in its capacity, which also makes the process cost effective and environmentally friendly.

\section{Introduction}

Marine oil spills occur frequently as a result of anthropogenic activities, such as oil rig drilling and oil tanker accidents, which may cause irreparable ecological impacts and devastating longterm environmental disasters. ${ }^{1}$ Various strategies, such as in situ burning, are being used for oil spill remediation; however, the gaseous products and particulates emitted through oil combustion pollute the atmosphere. ${ }^{2}$ The deployment of booms and skimmers confine the oil to a specific location for collection, but this method is inefficient in turbulent wavy surfaces, timeconsuming, laborious and requires a large number of equipment., ${ }^{3,4}$ Porous sorbent materials have recently attracted great attention owing to their ability to remove all traces of oil from spill sites. ${ }^{5}$ However, there are several challenges with these porous

\footnotetext{
${ }^{a}$ Department of Chemical and Biomolecular Engineering, Hong Kong University of Science and Technology, Clear Water Bay, Kowloon, Hong Kong. E-mail: keztluo@ ust.hk

${ }^{b}$ New York Center for Clean Water Technology, Stony Brook University, NY 11794, USA $\dagger$ Electronic supplementary information (ESI) available: FTIR analysis and full range XPS spectra of graphene oxide and graphene aerogel and Table S1-S3 are provided. See DOI: 10.1039/c7ra02886e
}

sorbents, including low uptake capacity and low sorption rate, which are yet to be overcome.,

Graphene, due to its extraordinary properties, has been used for numerous applications such as lithium ion batteries, supercapacitors, flexible electronics, sensors and composite materials. ${ }^{8}$ It is also a promising material for sorption applications due to its high surface area. However, the dispersion of pristine graphene in water is difficult which precludes its application in aqueous media. Therefore, graphene oxide (GO) is an alternative material to graphene that has facile aqueous solution processability due to the abundance of polar functional groups on its surface. ${ }^{9}$ For applications, especially those requiring high porosity such as contaminant removal from aqueous solution via sorption processes, the assembly of graphene oxide sheets into a three dimensional structure can be advantageous to form a material with a high surface area, enhanced porosity and high strength at very low density. ${ }^{10}$ This structure has been realized recently via the one step hydrothermal reduction of graphene oxide under controlled conditions ${ }^{\mathbf{1 1}}$ which has been used for lithium ion batteries and photocatalysis. ${ }^{\mathbf{1 2 - 1 4}}$ Unlike activated carbons and zeolites with micro- and/or mesoporous structures and high surface areas, ${ }^{15-17}$ aerogel sponges are mainly characterized by their high porosity and large macropores in addition to mesopores. Therefore, although activated carbons and zeolites employ 
electrostatic interaction or $\pi-\pi$ interactions to adsorb specific components, such as phenols and dyes, ${ }^{18-20}$ graphene aerogels are well-tailored for the absorption of nonpolar substances, such as hydrocarbons and oils. The great potential of graphene aerogels in oil sorption not only stems from their hydrophobicity, but also their large pore sizes which lead to facile oil diffusion into the bulk of the sorbent at high rates.

Some oil sorption studies using graphene based materials have been reported. For example, Bi et al. (2012) reported the synthesis of spongy graphene via a 24 hour hydrothermal reaction of a GO ammonia mixture for the adsorption of toxic solvents such as chloroform and toluene. ${ }^{21}$ Similarly, Zhao et al. (2012) used thiourea to fabricate graphene sponge for oil adsorption..$^{22}$ Bi et al. (2014) used freeze drying and soot treatment to prepare spongy graphene which improved its sorption capacities for different oils and organic solvents. ${ }^{23}$

In this work, an ultrahigh porosity hydrophobic graphene aerogel (GA) with a bimodal pore size distribution and meso and macropores is synthesized via a 2 hour hydrothermal reaction of only a pure GO solution and its sorption capacity is compared with its commercial counterparts and other porous materials in the literature. The rate of sorption is investigated in detail with a separate set of experiments for oils and organic solvents. Furthermore, the regeneration of the graphene aerogel is examined for multi-cycle operations in order to boost its economic viability and sustainability. Ultimately, the feasibility of fluoroalkyl silane grafting on the graphene aerogel surface is thoroughly examined with the aim of decreasing its surface energy as a result of the introduction of the C-F moiety so that GA can selectively uptake oil spilled on seawater with minimum sorption of water underneath in practical scenarios.

\section{Results and discussion}

\section{Morphology and chemical characterization}

Fig. 1 demonstrates the SEM image of the synthesized aerogel, where graphene sheets are randomly oriented and the interconnection of the stacked hierarchical macro-sized pores forms a three dimensional porous network with abundant voids, which is favorable for the uptake of molecules into the bulk of the material, i.e. absorption. Some literature hypothesize the potential of such materials with well-developed macroporous structures in the adsorption of pollutants from different media. ${ }^{24,25}$ Nevertheless, we believe that there is no evidence of any relationship between the presence of macropores and adsorption rate or intensity. The adsorption phenomenon is mainly associated with pores with sizes $<50 \mathrm{~nm}$ (micropores and mesopores), and not macropores. Since the detection of micro- and meso-structures is not feasible with SEM, the misconception of higher adsorption capacity by macroporous materials should be refrained. Nevertheless, welldeveloped interlinked hierarchical macropores can decrease the diffusion restrictions in the porous channels, resulting in a higher absorption rate and capacity. In this study, the SEM images illustrate that the size of the pores is in the range of 1-10 $\mu \mathrm{m}$, which is sufficiently large to diminish the pore blockage by oil and solvent molecules during the absorption process. The aforementioned characteristics of the aerogel, as evidenced by the SEM micrographs, render it a highly attractive material for applications requiring the absorption of contaminant molecules.

It is noteworthy that we do not discard the possibility of adsorption and the absorption phenomenon. We only suggest that it is not possible to conclude the influence of the adsorption process by only SEM images. Therefore, in order to quantitatively study the textural characteristics of the prepared aerogel, two types of widely applied analyses were adopted: mercury intrusion porosimetry for the determination of macropores $(>50 \mathrm{~nm})$ and mesopores $(2 \mathrm{~nm}<\mathrm{dp}<50 \mathrm{~nm})$ and nitrogen adsorption-desorption for the determination of micro$(<2 \mathrm{~nm})$ and mesopores. As illustrated in Fig. 2(e), the aerogel possesses a bimodal pore size distribution of macro- and mesopores, where the former has significantly higher pore volumes, which suggests the dominance of macropores in the aerogel (a)
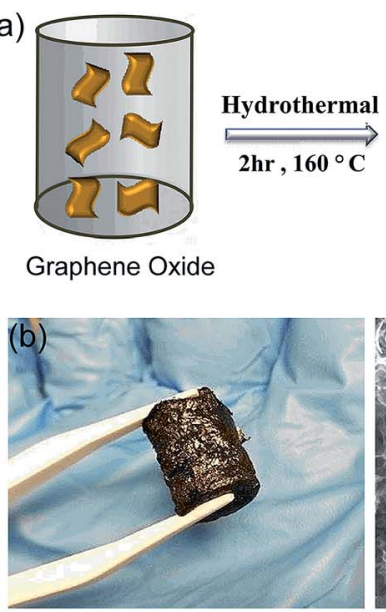

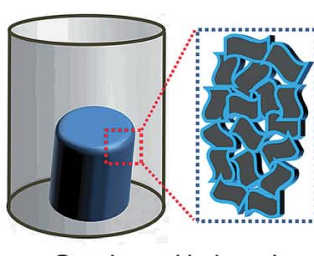

Graphene Hydrogel

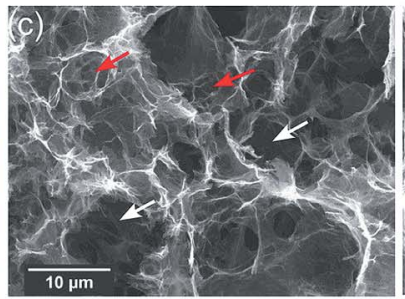

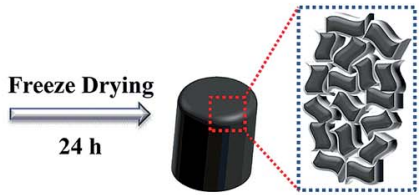

Graphene Aerogel

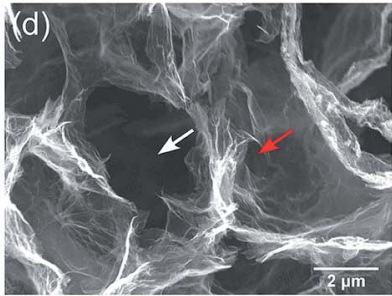

Fig. 1 Graphene aerogel synthesis and its morphology. (a) Schematic showing the experimental steps and synthetic conditions using graphene oxide as the precursor, (b) sample optical image illustrating the foam-type structure of the GA with good mechanical strength and (c and d) SEM images showing that graphene sheets are interconnected in a random fashion to form a three dimensional porous structure with pores of various sizes and shapes. 
structure. The macropores have a considerably wide range of sizes from $300 \mathrm{~nm}$ to over $10 \mu \mathrm{m}$, which implies the presence of a hierarchical porous structure and is in line with the observations from the SEM images. In contrast with the macropores, the mesopores have a much smaller pore size distribution in the range of 14-18 nm and much smaller pore volumes. The hierarchical pores can be hypothesized, but not solidly verified, to be in the form of large macropores in the outer surface of the aerogel interconnected with smaller macropores in the bulk of the material, which in turn, are linked to the smaller mesopores as side-branches of the larger pores. This unique hierarchical structure with a bimodal pore size distribution provides an accessible macropore structure which allows the diffusion of oils and organics into the bulk of the material at a high rate and ultimately their retention in the aerogel pore network. Although valuable information on the macropore size and distribution can be obtained through mercury intrusion porosimetry, the mesopores and micropores can be more accurately analyzed using the nitrogen adsorption-desorption method. As shown in Fig. 2(d), the $\mathrm{N}_{2}$ adsorption-desorption curve follows a type IV isotherm curve according to the IUPAC classification, which is characteristic of a mesoporous material. ${ }^{26}$ The low hysteresis loop area in the isotherm curve suggests the presence of a small volume of mesopores in the aerogel structure, which is in close agreement with the mercury intrusion porosimetry. The specific surface area of the graphene aerogel based on the BET model equation was determined to be $49.3 \mathrm{~m}^{2} \mathrm{~g}^{-1}$. Zhang et al. achieved a BET surface area of $11.8 \mathrm{~m}^{2} \mathrm{~g}^{-1}$ for their freeze dried graphene aerogel and suggested that employing a supercritical fluid drying instead of freeze drying would achieve higher specific surface areas. ${ }^{27}$ The surface area of graphene aerogels widely varies using various synthetic methods such as chemical reduction or hydrothermal self-assembly and synthetic conditions such as reaction time, temperature or GO concentration. We believe that the possible reason for a lower surface area is attributed to the aggregation of GO sheets during self-assembly. Nonetheless, despite the merit of increasing the BET surface area, the presence of macrospores is more favorable than mesopores in our specific application since absorption takes place by the diffusion of the fluid into the large macro-channels in the bulk of the material.

The C1s XPS spectra of the graphene oxide and graphene aerogel are shown in Fig. 2(a) and (b). For graphene oxide, four distinct peaks located at 284.6 (aromatic $\mathrm{C}-\mathrm{C}$ and $\mathrm{C}=\mathrm{C}$ ), 286.6 (epoxy or alkoxy C-O), 287.8 (carbonyl $\mathrm{C}=\mathrm{O}$ ) and $288.7 \mathrm{eV}$ (carboxyl $\mathrm{O}-\mathrm{C}=\mathrm{O}$ ) are identified on the surface of the GO. ${ }^{28}$ However, the C1s spectrum of the graphene aerogel exhibits markedly different peak intensities, where the aromatic $\mathrm{C}-\mathrm{C}$ and $\mathrm{C}=\mathrm{C}$ peak $(284.6 \mathrm{eV}$ ) is intensified and the peaks for the oxygen-containing functional groups exhibit significantly lower
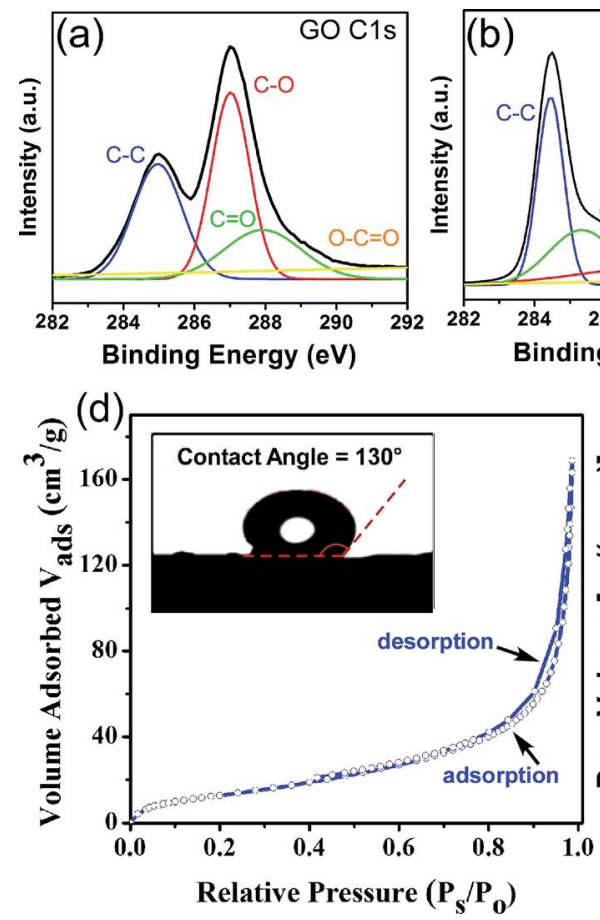
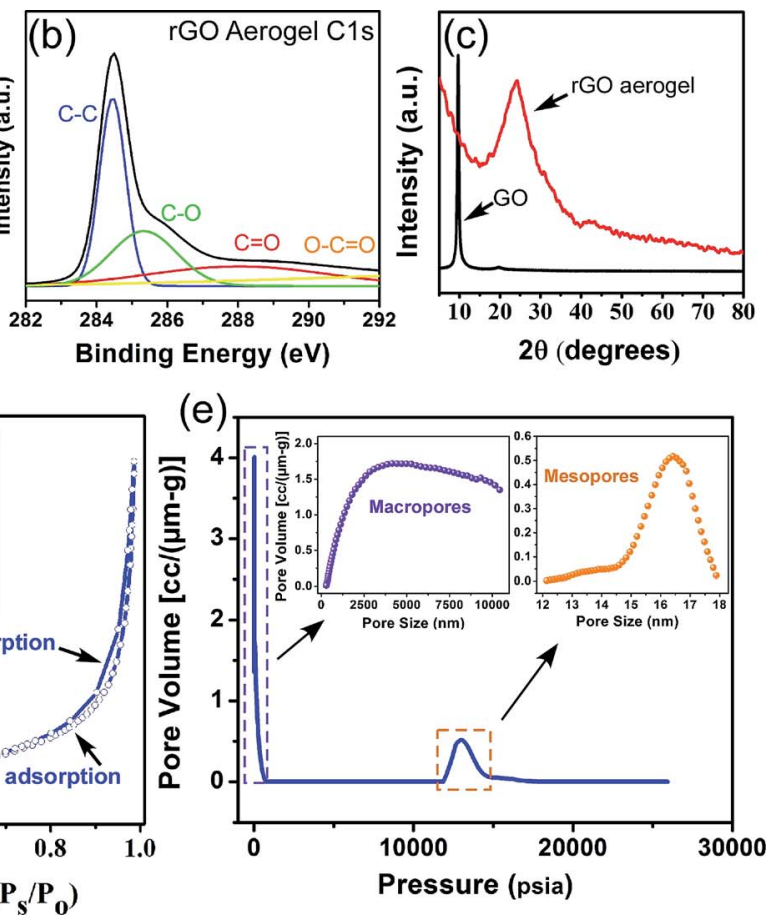

Fig. 2 Surface, structural and porous properties of the graphene aerogel. (a and b) C1s XPS spectra of the GA and GO samples showing that graphene oxide has different oxygen containing functional groups bonded to its surface; however, the graphene aerogel does not contain these functional groups in abundance as shown by the reduced intensity of these groups, (c) XRD results showing that the graphene aerogel has an amorphous structure in contrast to the crystalline structure of graphene oxide as it shows a sharp peak at around $10^{\circ}$, (d) $\mathrm{N}_{2}$ adsorptiondesorption isotherm of the GA showing the volume of $\mathrm{N}_{2}$ adsorbed at different relative pressures which is used to calculate the textural characteristics of the GA (inset shows the water contact angle of the graphene aerogel and its surface hydrophobicity) and (e) mercury intrusion of the graphene aerogel at different pressures (insets show the pore size distribution of the aerogel which suggests its hierarchical structure with mesopores and macropores of various sizes). 
intensities. Moreover, the peak area ratios of the oxygen to carbon $\left(A_{\mathrm{O}} / A_{\mathrm{C}}\right)$ obtained from the wide-range spectra (see Fig. $\mathrm{S} 1 \dagger)$ are 1.38 and 0.37 for GO and GA, respectively. The significantly lower $A_{\mathrm{O}} / A_{\mathrm{C}}$ for GA further confirms the efficient removal of oxygen-containing functional groups. The lower concentration of oxygenated surface functional groups is further verified by the FTIR analysis (see Fig. S2†). GO displays a series of absorption bands in the range of 3800 to $1000 \mathrm{~cm}^{-1}$, including a broad band at $3300 \mathrm{~cm}^{-1}$ for the hydroxyl groups, carbonyl bonds in both ketone and carboxylic acid groups at $1720 \mathrm{~cm}^{-1}$, aromatic $\mathrm{C}=\mathrm{C}$ bonds at $1581 \mathrm{~cm}^{-1}, \mathrm{C}-\mathrm{OH}$ stretching vibrations at $1404 \mathrm{~cm}^{-1}$ and epoxy or alkoxy $\mathrm{C}-\mathrm{O}$ groups at 1220 and $1080 \mathrm{~cm}^{-1}$. After hydrothermal reduction, the intensity of the hydroxyl $\mathrm{OH}$ groups decreased significantly, which suggests the removal of oxygen-containing groups from the surface of graphene. The reduction in the oxygen containing functional groups of the graphene aerogel, as elucidated by the XPS and FTIR techniques, is essential in applications requiring the separation of water insoluble compounds from aqueous media, since the diffusion of water molecules into the pores of the sorbent leads to the occupancy of the pores and thus, a reduction in the uptake capacity of the target water insoluble compounds. In order to examine its affinity for water, the wettability of the graphene aerogel surface was evaluated to around $130^{\circ}$, which indicates the water-repellent hydrophobic nature of the graphene aerogel surface. ${ }^{29}$ In addition, due to the very high oleophilic nature of the GA, an oil drop could not be formed on the GA surface for contact angle measurements. The high hydrophobicity and oleophilicity of GA validate it as an interesting material for the selective sorption of oil from aqueous media.

The XRD patterns illustrated in Fig. 2(c) show that the sharp peak at around $10^{\circ}$ corresponding to the crystalline structure of $\mathrm{GO}^{30}$ disappears upon the hydrothermal reaction, which represents the amorphous structure of the GA. The presence of a broad band at $25^{\circ}$ is attributed to the irregular structure of the GA and the random overlapping of the graphene sheets to form irregular network of pores, which is consistent with the structural observations by SEM.

\section{Sorption capacity and kinetics of the GA}

Drainage time is defined as the duration of time the GA is retained after the completion of the sorption in order to drain the oil molecules loosely attached to the outermost GA surface by cohesive and adhesive forces. This parameter is unfortunately either disregarded or not reported in most literature which may make the sorption capacities unreasonably high. ${ }^{25,31}$ Therefore, separate experiments were conducted to determine the appropriate drainage time of the GA using mineral and vegetable oils. The results shown in Fig. 3(a) reveal a very steep decrease in the uptake amount in the initial stage of the drainage for both oils. Therefore, the minimum drainage time of $1 \mathrm{~min}$ is required to ensure the removal of loosely-attached oil molecules from the GA surface. A drainage time of 2 min was considered in all subsequent experiments. Fig. 3(b) displays the sorption capacity of the graphene aerogel for several types of oils and organic solvents. The sorption capacity of the GA ranges from $58-70 \mathrm{~g} \mathrm{~g}^{-1}$ for oils and $44-68 \mathrm{~g} \mathrm{~g}^{-1}$ for organic solvents. Some previous studies ${ }^{21-23}$ reported similar or higher sorption capacities; however, GA was synthesized using different chemicals or synthetic methods and we reported a shorter hydrothermal synthetic method ( 2 hours) using pure GO. A detailed comparison of the synthesis and performance parameters of the present work with previously reported $3 \mathrm{D}$ graphene based sorbents is given in Table S3. $\dagger$

The physical and chemical properties of the sorbates are responsible for the difference in the uptake capacity of the sorbent material. ${ }^{32}$ Taking the organic solvent category as an example, a direct linear relationship between the GA uptake capacity and the solvent density is observed (see Fig. S3†). The weight-based uptake capacity is enhanced with an increase in the density of the sorbate, where hexane with the lowest density has the lowest sorption capacity and maximum uptake is achieved when DMF with the highest density is used as the sorbate. This trend is consistent with that reported in various other studies, such as the sorption of solvents by $\mathrm{Fe} / \mathrm{C}$ nanocomposites $^{33}$ and nanocellulose aerogels. ${ }^{34}$ If the obtained sorption capacities are transformed into the organic solvent volume (rather than weight) in unit weight of GA, the sorption
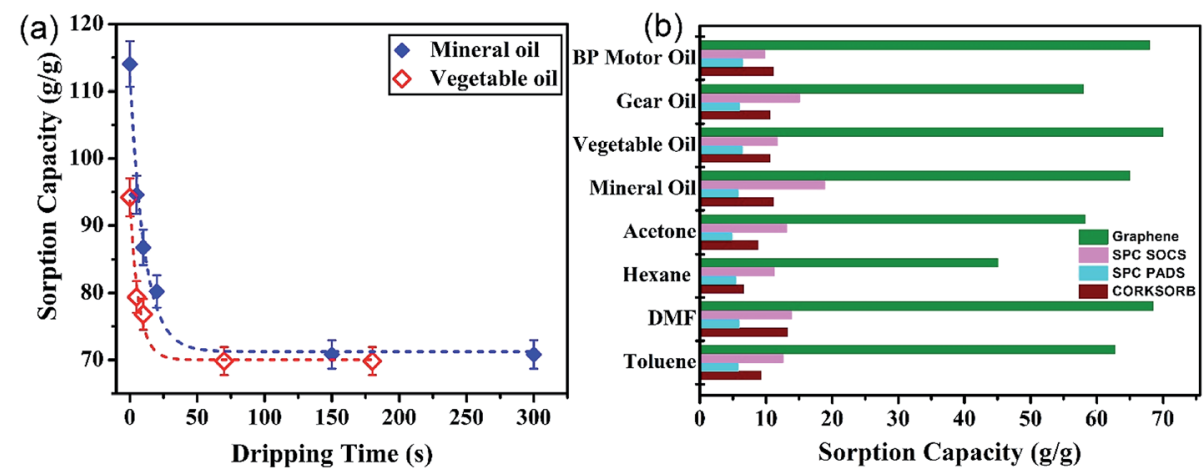

Fig. 3 Sorption capacity results of GA and the effect of drainage time. (a) GA sorption capacity decrease after the initial sorption of vegetable or mineral oil demonstrates that at least 1 minute of dripping or drainage after the oil sorption is required to represent the true sorption capacity of the graphene aerogel, (b) sorption capacities of the graphene aerogel for various oils and organic solvents and their comparison with commercial sorbents. The sorption capacities of the commercial sorbents Corksorb, Pads and SOCs are 6-14 $\mathrm{g} \mathrm{g}^{-1}, 5-7 \mathrm{~g} \mathrm{~g}^{-1}$ and $10-20 \mathrm{~g} \mathrm{~g}^{-1}$, respectively, which are much lower (more than 5 times) than that of the graphene aerogel with capacities of $40-70 \mathrm{~g} \mathrm{~g}^{-1}$ for various oils and organic solvents. 
capacity approaches an approximately constant value of $70 \mathrm{~mL}$ $\mathrm{g}^{-1}$. This finding is very interesting in the context of the sorption mechanism. Since the sorption capacity in terms of volume has an almost constant value, it can be concluded that the sorbent molecules are taken up in the bulk of the porous GA with a large volume of voids, which implies the dominance of the absorption mechanism rather than adsorption mechanism. This finding revamps the generic misconception that sorption using graphene or similar materials, such as synthetic carbon nanofiber films, ${ }^{24}$ porous carbon monoliths, ${ }^{35}$ silica monoliths ${ }^{36}$ or natural materials such as organoclays and raw cotton, ${ }^{37}$ proceeds via an adsorption mechanism. The sorption capacity of the GA is more impressive when comparisons with commonly-used commercial products under similar experimental conditions are made. As depicted in Fig. 5(b), the uptake capacity of the GA is much higher than the commercial sorbents used. The uptake capacity of the GA ranges from 40 to $70 \mathrm{~g} \mathrm{~g}^{-1}$, whereas Corksorb, SPC Pads and SPC SOCS, as the commercial sorbents, render sorption capacities in the ranges of 6-14 $\mathrm{g} \mathrm{g}^{-1}$, 5-7 $\mathrm{g} \mathrm{g}^{-1}$ and $10-20 \mathrm{~g} \mathrm{~g}^{-1}$, respectively, for the organic solvents and oils. Also, the GA sorption capacity was compared with that reported in literature, as summarized in Table S1 (ESI $\dagger$ ). It can be seen that only the CNT sponge has relatively comparable sorption capacities with that of the graphene aerogel. However, the facile preparation of the GA and its lower cost compared with the CNT sponge renders it a more attractive option for this application.
Although the high uptake capacity of the GA augments its potential for oil and organic solvent uptake commercially, several other characteristics of the GA, including sorption rate, recyclability and water affinity (selectivity), also need to be examined for its suitability in practical applications. The sorption kinetics of the GA for organic solvents is depicted in Fig. 4(a), where the removal of colored $n$-hexane by the GA is visually monitored. In this qualitative approach, the time-based fading of the color reflects the uptake rate of $n$-hexane. Here, the solvent uptake by the GA (only within $3 \mathrm{~s}$ ) is much faster than the sorption by the CNT sponge (125 $\mathrm{s}$ and $50 \mathrm{~s}$ for the sorption of octane and ethyl acetate, respectively). ${ }^{38}$ The high sorption rate of the GA is attributed to its hierarchical porous structure which facilitates the diffusion of molecules into its pores. Sorption rate is one of the critical factors in practical pollutant uptake applications, where most of the spreading occurs in the outset of the spillage. Similar experiments were performed for other organic solvents such as toluene and ethanol whose results reflect very fast sorption rates of a few seconds as well. An average uptake rate can be estimated using the sorption capacity and total time required to remove the colored solvent to reflect the sorption rate for each solvent. Considering the sorption capacity of the GA for $n$-hexane $\left(44 \mathrm{~g} \mathrm{~g}^{-1}\right)$ and the time required for the sorption process of $n$-hexane ( $4 \mathrm{~s})$, the average sorption rate was determined as $11 \mathrm{~g} \mathrm{~g}^{-1} \mathrm{~s}^{-1}$. Similarly, the sorption rates of the ethanol and toluene were determined as 22.9 and $17.7 \mathrm{~g} \mathrm{~g}^{-1} \mathrm{~s}^{-1}$, respectively (see Fig. 4(c)).
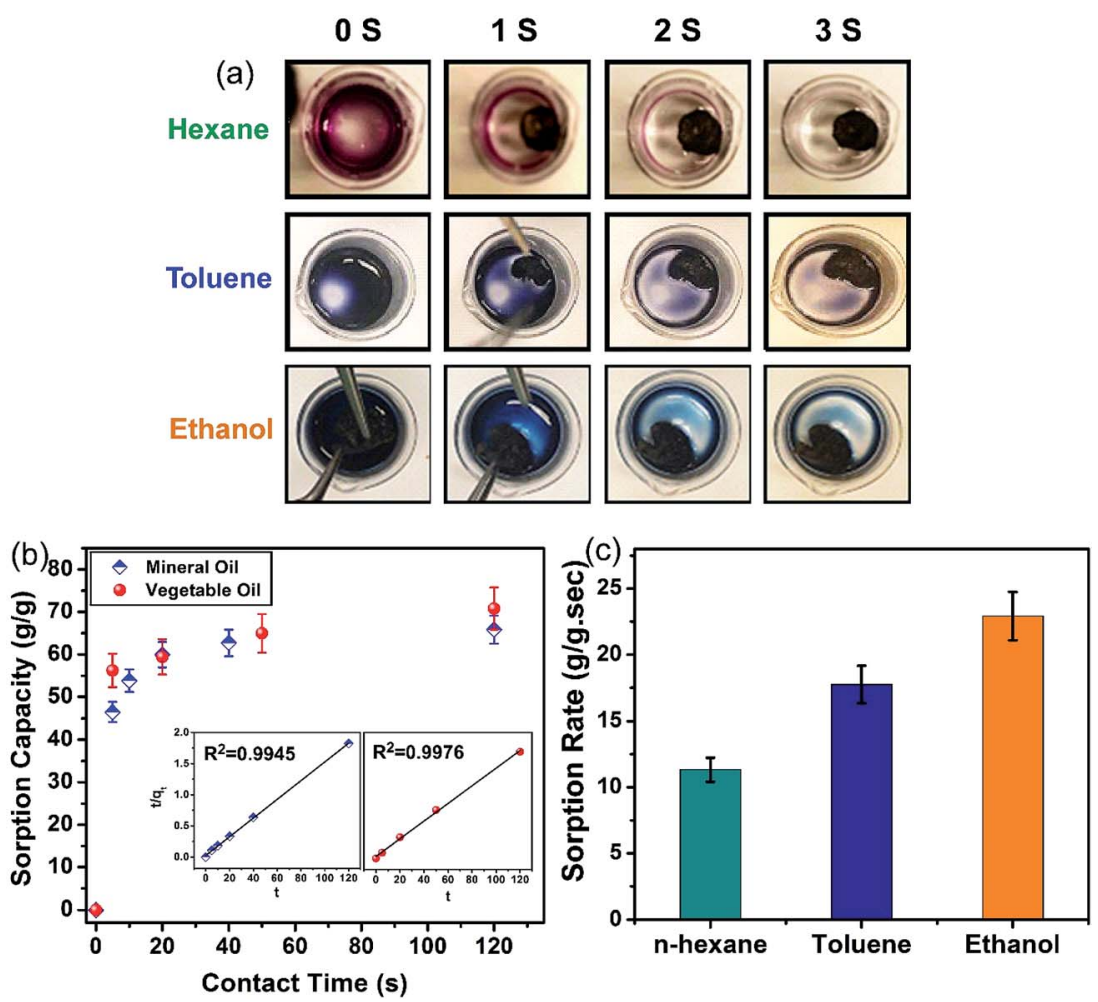

Fig. 4 Kinetics of the sorption of graphene aerogel. (a) Experimental system showing the sorption of $n$-hexane, toluene and ethanol by the graphene aerogel within 3 seconds, (b) oil uptake experiment using the graphene aerogel shows that the sorption reaches its maximum saturation level within 1 minute for mineral oil and vegetable oil (inset shows the linear fit of the experimental data to the pseudo second order kinetic model for both oils) and (c) average sorption rate of the organic solvents. 
In contrast to the organic solvent sorption, oil sorption uptake kinetics can be evaluated qualitatively. As illustrated in Fig. 4(b), the sorption rate of the oils is also very high, where the entire uptake process is completed in less than one minute. It is notable that $90 \%$ of the uptake for both oils is achieved in only $20 \mathrm{~s}$. The very fast uptake in the first few seconds is due to the lack of any diffusion barrier into the interior of the GA owing to the large size of its pores. However, after the macropores are filled, a comparatively longer time is required for the diffusion of the sorbed oil into the bulk of the GA with smaller macropores or even mesopores. It is shown that the experimental kinetic data best fits with the linearized form of the pseudo second order model (see inset of Fig. 4(a)) with correlation coefficients as high as 0.99 . The rate constants obtained using the pseudo second order model for the mineral oil and vegetable oil are 0.00476 and $0.00727 \mathrm{~s}^{-1}$, respectively. The model parameters obtained for the three different models are summarized in Table S2 (ESI $\dagger$ ). In previous studies ${ }^{\mathbf{2 1}, 22}$ there are some qualitative tests showing the fast uptake of oil by graphene aerogel, but in this study, the uptake kinetics of oils is quantitatively studied using a separately designed experiment and is verified by kinetic models. Moreover, the mechanism of oil sorption can be understood by this quantitative kinetic study as it reveals that $90 \%$ of oil uptake occurs within $20 \mathrm{~s}$.

\section{Recyclability and water sorption of GA}

Exploring the fate of the exhausted sorbent is a major challenge that is disregarded in some literature. Some researchers have proposed to burn oil-sorbed materials to utilize the calorific value of the recovered oil. ${ }^{34,38,39}$ However, this is not an appropriate method due to the unsustainability of the process and lack of any economic justification. Hence, employing a more sustainable approach for GA regeneration by removing the sorbed molecules from the pores of the GA and its reutilization is a more attractive and promising option. Fig. 5(a) shows the change in the oil sorption capacity of the GA and the material loss for several cycles and it can be seen that no material loss takes place during the regeneration process and that the capacity of the GA does not change and remains appreciably high even after 8 cycles of sorption-desorption. This is indeed very promising since the GA can be used almost infinitely in the oil sorption-regeneration process without the need for sorbent replacement. However, some sorbents lack this advantageous property. For instance, Deschamps et al. demonstrated that the oil sorption capacity of fresh cotton fiber is $20 \mathrm{~g} \mathrm{~g}^{-1}$, but after only 3 cycles of regeneration, its capacity decreases more than $30 \%$ to $14 \mathrm{~g} \mathrm{~g}^{-1} .^{40}$ Similarly, although the sorption capacity of polyurethane sponges was $19 \mathrm{~g} \mathrm{~g}^{-1}$ for the first cycle, it decreased to $7 \mathrm{~g} \mathrm{~g}^{-1}$ after 9 cycles. ${ }^{41}$ Also, although CNT sponge had a high sorption capacity of $100 \mathrm{~g} \mathrm{~g}^{-1}$ for vegetable oil, it could not retain its high capacity for many cycles, where its sorption capacity depleted to $20-40 \mathrm{~g} \mathrm{~g}^{-1}$ after 10 cycles of reuse. ${ }^{38}$ Unlike the regeneration of oil-sorbed GA using chemical methods, our regeneration is a thermal process in the case of the solvent-sorbed GA. Similar to the oil-sorbed GA, the GA exhibits an excellent recyclability, as shown in Fig. 5(a), where the sorption capacity of the aerogel did not show a significant decreasing trend even after several cycles of sorption and desorption and still maintained its high capacity. The amount of solvent that can be recovered is above $90 \%$ after each cycle.

Most of the studies on the uptake of oil by porous materials are carried out in idealistic conditions, where only oil (in the absence of water) is used as the sorption medium. ${ }^{\mathbf{3 8 , 4 2 , 4 3}}$ Nevertheless, in practical scenarios, oil spreads as a thin layer on water bodies, thus it is important to simulate real conditions and determine the uptake efficiency of the sorbent in the presence of water. In seawater, oil slick of few micrometers is formed whose thickness is used to determine the volume of the oil spill. Therefore, to simulate the oil spill sea conditions, different amounts of the oil was spilt on a fixed surface area of water and the sorption capacity of the GA was evaluated. Fig. 5(b) shows that the GA also takes up water along with oil. Although the water sorption amount of the GA is low, the oil sorption amount, even in the presence of water, is higher than its commercial counterparts. However, this can be considered as an undesirable phenomenon as it reduces the sorption capacity of the GA. The water sorption of the GA is mainly due to the presence of some functional groups (although in a low concentration) on the GA surface. As illustrated in Fig. 5(c),
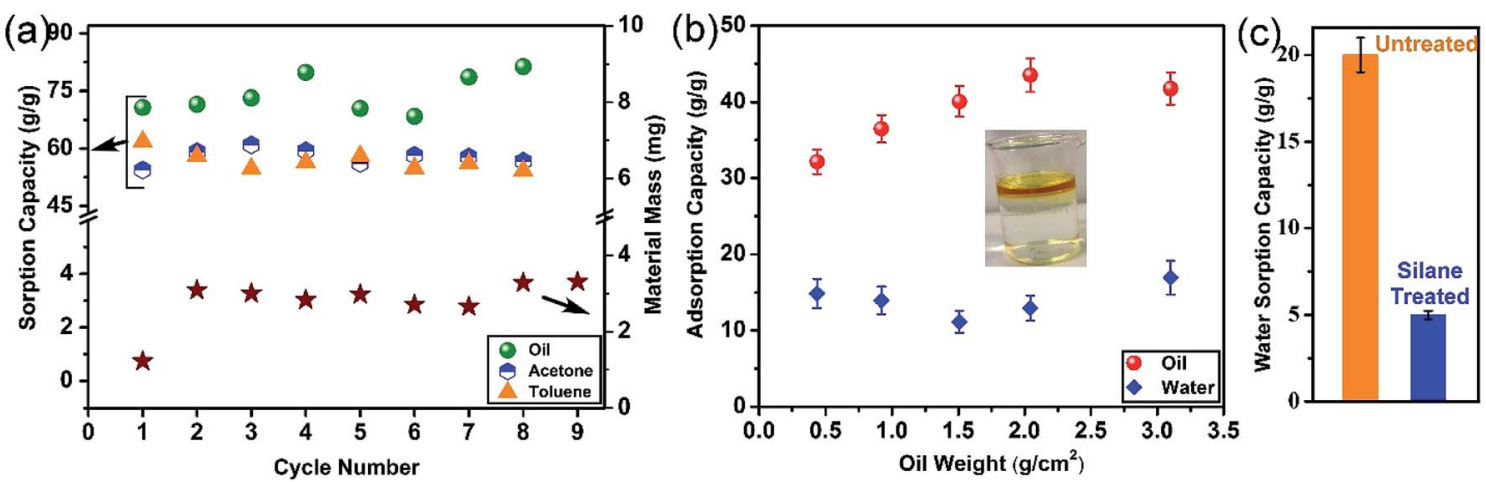

Fig. 5 Multicycle recyclability test and water sorption capacity of the GA. (a) Thermal and chemical regeneration of the organic solvent-sorbed GA and oil-sorbed GA, respectively, and their re-use for several cycles without much change in sorption capacity, (b) different concentrations of oil on water and the corresponding uptake of oil and water by the GA and (c) water sorption capacity of GA before and after silane functionalization showing a drastic reduction in the water uptake. 
although the water uptake of the GA is around $20 \mathrm{~g} \mathrm{~g}^{-1}$, silanegrafted GA has a water uptake as low as $5 \mathrm{~g} \mathrm{~g}^{-1}$. The full range XPS spectra of silane functionalized GA in Fig. S5† shows prominent fluorine peak hence this low water affinity is attributed to the lower surface energy of the GA due to the presence of C-F groups which can mask the moieties with higher surface energies and thus results in the diminution of the water sorption.

It should be noted that silane modification of graphene aerogel does not change its morphological properties such as SSA and porous structures. However, silane treatment makes the surface water repellent as indicated by the contact angle measurements, which makes the oil sorption process more effective and efficient.

Overall, it has been demonstrated in our work that this GA with a unique hierarchal bimodal meso-macro porous structure overcomes most of the challenges associated with conventional sorbent materials such as insufficient sorption capacity, low uptake rate, non-recyclability, and high water uptake rendering it useful for practical spills of petroleum liquids in seawater. Moreover, effort has been made to study in depth the kinetics of sorption the GA for various oils and organic solvents and to understand mechanism behind very high sorption rate of the GA through detailed characterization of its unique porous structure.

\section{Experimental}

\section{Chemicals}

Expandable graphite flakes (GRAFGUARD® 160-50N) with a typical expansion volume of $250 \mathrm{~cm}^{3} \mathrm{~g}^{-1}$ at $600{ }^{\circ} \mathrm{C}$ and mean particle size of around $350 \mu \mathrm{m}$ were purchased from Graftech Hong Kong Limited. Microwave heating was used to form expanded graphite before the experiments. All other chemicals of analytical grade were purchased from Sigma-Aldrich, Hong Kong, and were used without further purification. Kaydol White Mineral Oil was supplied by Sonneborn. Vegetable oil with $50.5 \%$ pomace olive oil as its main constituent was purchased from a local market in Hong Kong. The other oils used were engine oil Visco 5000 of British Petroleum (BP) and gear oil 80W-90 of Americo Lubricants Ltd. Three different commercial sorbents were used in this research. Thermally-treated hydrophobic granular Corksorb product was purchased from Amorim Environmental Systems, Portugal and polypropylene SOCs and Pads were obtained from Brady Corporation Hong Kong Limited.

\section{Material preparation}

Preparation of graphene oxide (GO). Graphene oxide was prepared via a modified Hummers method ${ }^{\mathbf{4 4}}$ where expansion of graphite was carried out prior to the intercalation or oxidation stage by microwave heating for few seconds. Subsequently, $1 \mathrm{~g}$ microwave-treated expanded graphite, $5 \mathrm{~g} \mathrm{~K} \mathrm{~K}_{8} \mathrm{O}_{8}$ and $5 \mathrm{~g} \mathrm{P}_{2} \mathrm{O}_{5}$ were added to a round bottom flask containing $30 \mathrm{~mL}$ concentrated sulfuric acid. The desired reaction took place by thoroughly stirring the mixture at $90{ }^{\circ} \mathrm{C}$ for $4.5 \mathrm{~h}$ using an oil bath as the heating medium. The oxidized product was then filtered using a 0.2 micron Nylon Millipore filter and washed with DI water using a vacuum-assisted filtration setup until a $\mathrm{pH}$ level of 5.5 was achieved. This acid intercalated sample was dried in an oven at $60{ }^{\circ} \mathrm{C}$ overnight. In the next stage, the dried sample was introduced into a $250 \mathrm{~mL}$ round bottom flask containing $150 \mathrm{~mL}$ concentrated sulfuric acid and $30 \mathrm{~g} \mathrm{KMnO}$ was gradually added it. The mixture was stirred and reacted for $4 \mathrm{~h}$ at $35{ }^{\circ} \mathrm{C}$. Subsequently, the mixture was added to $1 \mathrm{~L} \mathrm{DI}$ water and stirred for another $2 \mathrm{~h}$. In the last stage, $50 \mathrm{~mL} \mathrm{30 \%}$ $\mathrm{H}_{2} \mathrm{O}_{2}$ was added dropwise to the mixture. The purple color of the mixture changed to bright yellow at the end of this step. The mixture was further stirred for $2 \mathrm{~h}$ and allowed to settle for $24 \mathrm{~h}$. Ultimately, the supernatant was discarded and the product at the bottom (graphene oxide, GO) was washed and centrifuged first with $1 \mathrm{~L} 10 \% \mathrm{HCl}$ solution and then with excess DI water until neutrality was achieved.

Preparation of graphene aerogel (GA). The graphene aerogel was prepared via a hydrothermal method where $10 \mathrm{~mL}$ of graphene oxide solution $\left(>2 \mathrm{mg} \mathrm{mL} \mathrm{m}^{-1}\right.$ ) was placed inside a Teflonlined autoclave reactor and heated to $160{ }^{\circ} \mathrm{C}$ for $2 \mathrm{~h}$. The prepared graphene hydrogel was freeze-dried for $24 \mathrm{~h}$ after which the porous graphene aerogel (GA) was obtained.

Fluoroalkyl silane grafting onto the GA surface. In the first step, a solution was prepared by dissolving $200 \mu \mathrm{L}$ $1 H, 1 H, 2 H, 2 H$-perfluorooctyltriethoxysilane (POTS) in $15 \mathrm{~mL}$ hexane in a glass vial. $1 \mathrm{~mL}$ water was added afterwards and the solution was stirred for 15 min to fully hydrolyze the POTS. In the second step, the graphene aerogel was immersed into the solution and stirred for $12 \mathrm{~h}$ to allow the surface functional groups of the GA to react with the hydrolyzed POTS. The fluorosilane-treated aerogel was then heated at $80{ }^{\circ} \mathrm{C}$ overnight and the resultant material was denoted as FS-GA.

\section{Characterization}

The surface hydrophobicity of the graphene aerogel was determined using a DIGIDROP contact angle meter (GBX, France). Attenuated total reflectance Fourier transform infrared (ATRFTIR) spectra were recorded on a Bio-Rad FTS 6000 spectrometer in the wavelength range of $4000-500 \mathrm{~cm}^{-1}$ to determine the surface functional groups of the prepared materials. X-ray diffraction analysis (XRD, PW1830, Philips) with $\mathrm{Cu} \mathrm{K} \alpha$ radiation $(\lambda=1.5406 \AA)$ was used to study the crystal structure of the materials. The morphology of the materials was investigated on a scanning electron microscope (SEM, JEOL 6700F). X-ray photoelectron (XPS) analysis was carried out using an XPSPHI5600 system equipped with a monochromatic $\mathrm{Al} \mathrm{K \alpha}$ source to understand the chemical bonding states of the surface elements in the materials. The textural properties of the materials were analyzed on a Beckman Coulter SA3100 surface area analyzer using the nitrogen adsorption-desorption method at $77 \mathrm{~K}$. The Brunauer-Emmett-Teller (BET) and the Barrett-Joyner-Halenda (BJH) equations were employed to evaluate the specific surface area and pore size distribution of the materials, respectively. The samples were outgassed at $120{ }^{\circ} \mathrm{C}$ prior to the analysis in order to remove all the moisture and adsorbed gases on the materials. 


\section{Sorption experiments}

Sorption capacity. Accurately-weighed graphene aerogel samples (GA, $5 \mathrm{mg}$ ) were immersed in different types of sorbates (oils and organic solvents) for 2 min (contact time). Then, the samples were gently taken out and allowed to drain for $2 \mathrm{~min}$ in order to remove the molecules loosely attached to the outermost surface of the sorbent by cohesive and adhesive forces (and not as a result of diffusion into the bulk of the material). Then, the GA samples were weighed and the equilibrium sorption capacity of the GA was determined as the amount of sorbate taken up on a unit mass of dry GA, as follows:

$$
Q_{\mathrm{e}}=\frac{W_{\mathrm{f}}-W_{\mathrm{i}}}{W_{\mathrm{i}}}
$$

where, $Q_{\mathrm{e}}$ is the sorption capacity of the graphene aerogel and $W_{\mathrm{i}}$ and $W_{\mathrm{f}}$ are the initial and final weights of the samples before and after the oil sorption, respectively. An identical approach was assumed to test the sorption efficiency of the commercial sorbents.

Sorption kinetics. Two distinct approaches were attempted to determine the sorption rate of the organic solvents and oils. For the sorption kinetics of organic solvents, the sorbates were first stained with an organic dye (Sudan Black B, $1 \mathrm{mg} \mathrm{mL} \mathrm{mL}^{-1}$ ) and then added on water to create artificial seawater conditions in a visible manner. For the sorption kinetics of oil, graphene aerogel samples $(5 \mathrm{mg}$ ) were placed in separate containers with equal amounts of oil for different contact times and the weight of each GA sample was measured after 2 min of drainage. The uptake capacity of the sorbent at time $t$ was determined as follows:

$$
Q_{t}=\frac{W_{t}-W_{\mathrm{i}}}{W_{\mathrm{i}}}
$$

where, $Q_{t}$ is the sorption capacity of the GA at specific time $t, W_{\mathrm{i}}$ is the initial weight of the sorbent and $W_{t}$ is the weight of the sorbent at time $t$. The kinetic data were analyzed using several kinetic models such as the pseudo first and second order models which are mathematically represented by the following equations:

$$
\begin{gathered}
\ln \left(q_{\mathrm{e}}-q_{t}\right)=\ln \left(q_{\mathrm{e}}\right)-k_{1} t \\
\frac{t}{q_{t}}=\frac{1}{k_{2} q_{\mathrm{e}}^{2}}+\frac{t}{q_{\mathrm{e}}}
\end{gathered}
$$

where, $q_{\mathrm{e}}$ is the maximum sorption capacity of the graphene aerogel, $q_{t}$ is the sorption amount of the graphene aerogel at equilibrium time $t$, and $k_{1}$ and $k_{2}$ are rate constants of the pseudo first and second order models, respectively. The intraparticle diffusion model was also applied to study the oil uptake kinetics by graphene aerogel:

$$
\mathrm{q}_{t}=k_{\mathrm{i}} t^{0.5}+\mathrm{c}
$$

where, $k_{\mathrm{i}}$ is the intra-particle diffusion rate constant $\left(\mathrm{g} \mathrm{g}^{-1}\right.$ $\left.\min ^{-0.5}\right)$.

Recyclability test. To regenerate the aerogel for organic solvents, the aerogel with sorbed organic solvent was heated above the boiling point of the solvent and the as-dried aerogel was used in the next sorption cycle. The weight change after each cycle reflects the regeneration ability of the GA. For the oilsorbed GA, the aerogel was submerged into $n$-hexane which diffused into the pores of the GA and washed the oil away. Subsequently, the aerogel was dried in an oven and used for the subsequent sorption cycles. The capacities of the GA in each cycle were determined as described earlier.

Water sorption and selectivity. The water sorption capacities of the GA and silane-modified GA were evaluated by their immersion in pure water and monitoring their weight change. Moreover, the selectivities of the graphene aerogel samples were determined by introducing different amounts of oil onto the water surface and carrying out the aforementioned sorption tests.

\section{Acknowledgements}

This project is supported by the Research Grant Council of Hong Kong SAR (project no. 623512 and DAG12EG05). Mr. Riaz appreciates financial support from Higher Education Commission (HEC) of Pakistan. Technical assistance from the Materials Characterization and Preparation Facilities is greatly appreciated.

\section{References}

1 T. J. Crone and M. Tolstoy, Magnitude of the 2010 Gulf of Mexico Oil Leak, Science, 2010, 330, 634.

2 J. V. Mullin and M. A. Champ, Introduction/Overview to In Situ Burning of Oil Spills, Spill Sci. Technol. Bull., 2003, 8, 323-330.

3 A. A. Al-Majed, A. R. Adebayo and M. E. Hossain, A Sustainable Approach to Controlling Oil Spills, J. Environ. Manage., 2012, 113, 213-227.

4 R. Schulze. Oil Spill Response Performance Review of Skimmers, ASTM, 1998.

5 J. Rogowska and J. Namie. Reviews of Environmental Contamination and Toxicology Volume 206, Reviews of Environmental Contamination and Toxicology, Springer, New York, 2010, vol. 206.

6 M. O. Adebajo, R. L. Frost, J. T. Kloprogge, O. Carmody and S. Kokot, Porous Materials for Oil Spill Cleanup: A Review of Synthesis and Absorbing Properties, J. Porous Mater., 2003, 10, 159-170.

7 A. Li, H.-X. Sun, D.-Z. Tan, W.-J. Fan, S.-H. Wen, X.-J. Qing, G.-X. Li, S.-Y. Li and W.-Q. Deng, Superhydrophobic Conjugated Microporous Polymers for Separation and Adsorption, Energy Environ. Sci., 2011, 4, 2062.

8 K. S. Novoselov, V. I. Fal'ko, L. Colombo, P. R. Gellert, M. G. Schwab and K. Kim, A Roadmap for Graphene, Nature, 2012, 490, 192-200.

9 H.-P. Cong, J.-F. Chen and S.-H. Yu, Graphene-Based Macroscopic Assemblies and Architectures: An Emerging Material System, Chem. Soc. Rev., 2014, 43, 7295-7325.

10 M. A. Worsley, T. Y. Olson, J. R. I. Lee, T. M. Willey, M. H. Nielsen, S. K. Roberts, P. J. Pauzauskie, J. Biener, 
J. H. Satcher and T. F. Baumann, High Surface Area, Sp 2Cross-Linked Three-Dimensional Graphene Monoliths, J. Phys. Chem. Lett., 2011, 2, 921-925.

11 Y. Xu, K. Sheng, C. Li and G. Shi, Self-Assembled Graphene Hydrogel via One-Step Hydrothermal Process, ACS Nano, 2010, 4, 4324-4330.

12 B. Qiu, M. Xing, J. Zhang, B. Qiu, M. Xing and J. Zhang, Mesoporous $\mathrm{TiO}_{2}$ Nanocrystals Grown In-Situ on Graphene Aerogels for High Photocatalysis and Lithium Ion Batteries, J. Am. Chem. Soc., 2014, 136, 5852-5855.

13 D. A. Reddy, J. Choi, S. Lee, R. Ma and T. K. Kim, SelfAssembled Macro Porous ZnS-graphene Aerogels for Photocatalytic Degradation of Contaminants in Water, $R S C$ Adv., 2015, 5, 18342-18351.

14 Z. Chen, H. Li, R. Tian, H. Duan, Y. Guo, Y. Chen, J. Zhou, C. Zhang, D. Roberto and H. Liu, Three Dimensional Graphene Aerogels as Binder-Less, Freestanding, Elastic and High-Performance Electrodes for Lithium-Ion Batteries, Sci. Rep., 2016, 6, 27365.

15 P. Hadi, C. Ning, W. Ouyang, C. S. K. Lin, C.-W. Hui and G. McKay, Conversion of an Aluminosilicate-Based Waste Material to High-Value Efficient Adsorbent, Chem. Eng. J., 2014, 256, 415-420.

16 P. Hadi, M. Xu, C. Ning, C. Sze Ki Lin and G. A. McKay, Critical Review on Preparation, Characterization and Utilization of Sludge-Derived Activated Carbons for Wastewater Treatment, Chem. Eng. J., 2015, 260, 895-906.

17 P. Hadi, J. Barford and G. Mckay, Toxic Heavy Metal Capture Using a Novel Electronic Waste-Based Material - Mechanism, Modeling and Comparison, Environ. Sci. Technol., 2013, 47, 8248-8255.

18 P. Hadi, K. Y. Yeung, J. Barford, K. J. An and G. McKay, Significance of Microporosity on the Interaction of Phenol with Porous Graphitic Carbon, Chem. Eng. J., 2015, 269, 20-26.

19 P. Hadi, K. Ying, J. Barford, K. Jin and G. Mckay, Significance of "effective" Surface Area of Activated Carbons on Elucidating the Adsorption Mechanism of Large Dye Molecules, J. Environ. Chem. Eng., 2015, 3, 1029-1037.

20 P. Hadi, J. Guo, J. Barford and G. McKay, Multilayer Dye Adsorption in Activated Carbons-Facile Approach to Exploit Vacant Sites and Interlayer Charge Interaction, Environ. Sci. Technol., 2016, 50, 5041-5049.

21 H. Bi, X. Xie, K. Yin, Y. Zhou, S. Wan, L. He, F. Xu, F. Banhart, L. Sun and R. S. Ruoff, Spongy Graphene as a Highly Efficient and Recyclable Sorbent for Oils and Organic Solvents, Adv. Funct. Mater., 2012, 22, 4421-4425.

22 J. Zhao, W. Ren and H.-M. Cheng, Graphene Sponge for Efficient and Repeatable Adsorption and Desorption of Water Contaminations, J. Mater. Chem., 2012, 22, 20197.

23 H. Bi, X. Xie, K. Yin, Y. Zhou, S. Wan, R. S. Ruoff and L. Sun, Highly Enhanced Performance of Spongy Graphene as an Oil Sorbent, J. Mater. Chem. A, 2014, 2, 1652-1656.

24 H. Liu, C.-Y. Cao, F.-F. Wei, P.-P. Huang, Y.-B. Sun, L. Jiang and W.-G. Song, Flexible Macroporous Carbon Nanofiber Film with High Oil Adsorption Capacity, J. Mater. Chem. A, 2014, 2, 3557 .
25 S. Huang and J. Shi, Monolithic Macroporous Carbon Materials as High-Performance and Ultralow-Cost Sorbents for Efficiently Solving Organic Pollution, Ind. Eng. Chem. Res., 2014, 53, 4888-4893.

26 P. Hadi, C. Ning, J. D. Kubicki, K. Mueller, J. W. Fagan, Z. Luo, L. Weng and G. McKay, Sustainable Development of a Surface-Functionalized Mesoporous Aluminosilicate with Ultra-High Ion Exchange Efficiency, Inorg. Chem. Front., 2016, 3, 502-513.

27 X. Zhang, Z. Sui, B. Xu, S. Yue, Y. Luo, W. Zhan and B. Liu, Mechanically Strong and Highly Conductive Graphene Aerogel and Its Use as Electrodes for Electrochemical Power Sources, J. Mater. Chem., 2011, 21, 6494.

28 D. C. Marcano, D. V. Kosynkin, J. M. Berlin, A. Sinitskii, Z. Sun, A. Slesarev, L. B. Alemany, W. Lu and J. M. Tour, Improved Synthesis of Graphene Oxide, ACS Nano, 2010, 4, 4806-4814.

29 J. Rafiee, M. A. Rafiee, Z.-Z. Yu and N. Koratkar, Superhydrophobic to Superhydrophilic Wetting Control in Graphene Films, Adv. Mater., 2010, 22, 2151-2154.

30 A. M. Dimiev and J. M. Tour, Mechanism of Graphene Oxide Formation, ACS Nano, 2014, 8, 3060-3068.

31 Z.-Y. Wu, C. Li, H.-W. Liang, J.-F. Chen and S.-H. Yu, Ultralight, Flexible, and Fire-Resistant Carbon Nanofiber Aerogels from Bacterial Cellulose, Angew. Chem., Int. Ed. Engl., 2013, 52, 2925-2929.

32 V. Rao, N. D. Hegde and H. Hirashima, Absorption and Desorption of Organic Liquids in Elastic Superhydrophobic Silica Aerogels, J. Colloid Interface Sci., 2007, 305, 124-132.

33 Y. Chu and Q. Pan, Three-Dimensionally Macroporous Fe/C Nanocomposites as Highly Selective Oil-Absorption Materials, ACS Appl. Mater. Interfaces, 2012, 4, 2420-2425.

34 J. T. Korhonen, M. Kettunen, R. H. A. Ras and O. Ikkala, Hydrophobic Nanocellulose Aerogels as Floating, Sustainable, Reusable, and Recyclable Oil Absorbents, ACS Appl. Mater. Interfaces, 2011, 3, 1813-1816.

35 G. Tao, L. Zhang, Z. Hua, Y. Chen, L. Guo, J. Zhang, Z. Shu, J. Gao, H. Chen, W. Wu, et al., Highly Efficient Adsorbents Based on Hierarchically Macro/mesoporous Carbon Monoliths with Strong Hydrophobicity, Carbon, 2014, 66, 547-559.

36 T. Sun, H. Fan, Z. Wang and Z. Wu, Hydrophobic Monolithic Silica with Abundant Pore as Efficient Adsorbent for Organic Contaminants Removal, J. Mater. Sci., 2013, 48, 6713-6718.

37 O. Carmody, R. Frost, Y. Xi and S. Kokot, Selected Adsorbent Materials for Oil Spill Cleanup A Thermoanalytical Study, J. Therm. Anal. Calorim., 2008, 91, 809-816.

38 X. Gui, H. Li, K. Wang, J. Wei, Y. Jia, Z. Li, L. Fan, A. Cao, H. Zhu and D. Wu, Recyclable Carbon Nanotube Sponges for Oil Absorption, Acta Mater., 2011, 59, 4798-4804.

39 D. P. Hashim, N. T. Narayanan, J. M. Romo-Herrera, D. A. Cullen, M. G. Hahm, P. Lezzi, J. R. Suttle, D. Kelkhoff, E. Muñoz-Sandoval, S. Ganguli, et al., Covalently Bonded Three-Dimensional Carbon Nanotube Solids via Boron Induced Nanojunctions, Sci. Rep., 2012, 2, 363. 
40 G. Deschamps, H. Caruel, M.-E. Borredon, C. Bonnin and C. Vignoles, Oil Removal from Water by Selective Sorption on Hydrophobic Cotton Fibers, Environ. Sci. Technol., 2003, 37, 1013-1015.

41 Q. Zhu, Q. Pan and F. Liu, Facile Removal and Collection of Oils from Water Surfaces through Superhydrophobic and Superoleophilic Sponges, J. Phys. Chem. C, 2011, 115, 17464-17470.
42 V. Singh, R. J. Kendall, K. Hake and S. Ramkumar, Crude Oil Sorption by Raw Cotton, Ind. Eng. Chem. Res., 2013, 52, 62776281.

43 X. Zhou, F. Wang, Y. Ji and J. Wei, Fabrication of Unidirectional Diffusion Layer onto Polypropylene Mat for Oil Spill Cleanup, Ind. Eng. Chem. Res., 2015, 54, 1177211778.

44 Z. Luo, Y. Lu, L. A. Somers and A. T. C. Johnson, High Yield Preparation of Macroscopic Graphene Oxide Membranes, $J$. Am. Chem. Soc., 2009, 131, 898-899. 